\title{
EXACT INTEGRATION OF A NONLINEAR MODEL OF STEADY HEAT CONDUCTION/RADIATION IN A WIRE WITH INTERNAL POWER
}

\author{
GIOVANNI M. SCARPELLO, ARSEN PALESTIN AND DANIELE RITELLI
}

Communicated by Mauro Spera

\begin{abstract}
The paper treats in one dimensional mixed heat transfer problem of steady conduction and radiation in a wire with internal source. We are led to a Cauchy problem consisting of a second order nonlinear ordinary differential equation. A special integrable case with two non independent left boundary conditions requires a hyperelliptic integral, for which a representation theorem has been established through the Gauss hypergeometric function ${ }_{2} F_{1}$. The relevant steady solution is then found to grow monotonically with the distance from boundary, up to a certain limiting position where it suddenly jumps unbounded.
\end{abstract}

\section{Introduction}

Conduction, namely the flow of thermal energy through solid bodies, was modelled by Jean B. Fourier (1768-1830) who first inquired into the general principles of it. Throughout his Théorie analytique de la chaleur (1822), he established a partial differential equation (PDE) for analyzing the temperature distribution within a conducting body. His analytical conduction theory disregards the molecular structure of a body and thinks of it as a continuum, but after Fourier it has been understood that-on the contrary- conduction is actually caused by particle collisions. His linear PDE, in one dimensional geometry, is

$$
\rho c_{p} \frac{\partial T}{\partial t}(t, x)=\chi \frac{\partial^{2} T}{\partial x^{2}}(t, x)
$$

where the material data are: thermal conductivity $\chi$, specific heat capacity $c_{p}$ and volumetric density $\rho$. As far as it concerns the spatial effects, the PDE has to be solved with suitable boundary conditions (BC).

Transient problems $(\partial T / \partial t \neq 0)$ will also need initial conditions (IC) on $T$ for every position in the system: the PDE is parabolic and heat propagates at infinite 
speed. Several investigators, (see e.g. [4] page 865) pointed out that transient heat really flows with a finite heat propagation speed, and then the adequate PDE governing it should be more complicated and of hyperbolic type (telegraph PDE), see [2]. Nevertheless the instantaneous unsteady equation is sufficient for most applications, and can be enlarged to include an inner source $q_{v}=$ constant, or a forcing function of $t$

$$
\rho c_{p} \frac{\partial T}{\partial t}(t, x)=\chi \frac{\partial^{2} T}{\partial x^{2}}(t, x)+q_{v}(t)
$$

where each term is a specific power, namely energy for unit time and volume. Such is the case of a radioactive source, whose power generation depends on the thermal yield of each transition multiplied by decaying nuclei number ( $a c$ tivity) for unit time and volume. Moreover, faced with very high temperatures, a realistic model should take into account radiation by or to the body and which propagates even in a lack of medium. The radiated power by a $T$-hot body in a $T_{0}$-environment is ruled by the Stefan-Boltzmann law of the fourth powers of temperatures. In such a way, we do not have a linear conduction equation any longer, but a mixed conduction/radiation nonlinear PDE

$$
\rho c_{p} \frac{\partial T}{\partial t}(t, x)=\chi \frac{\partial^{2} T}{\partial x^{2}}(t, x)-\alpha\left(T^{4}(t, x)-T_{0}^{4}\right)+q_{v}
$$

a much more difficult problem.

It has not to be confused with a conduction with radiative boundary condition. For example, for an infinitely long $R$-cylinder the radial conduction is modelled by a linear ODE in $T(r)$ and in such case the radiation towards the surroundings will involve only $T(R)$ with a nonlinear condition on it. In our paper we will consider a one dimensional, steady, thermal, axial conduction with simultaneous radiation, which we will integrate with the help of the hypergeometric function.

\section{The Steady Thermal System and its Nonlinear ODE}

Our system consists of a homogeneous, indefinite wire of radius $R$, whose material is characterized by the constants $\rho, c_{p}, \chi$ and having a cross section of area $\omega$ and a perimeter $p$.

The temperature equation along a thin wire where a constant electric current is flowing in, was given in 1872 by Verdet

$$
\frac{\partial T}{\partial t}(t, x)=k \frac{\partial^{2} T}{\partial x^{2}}(t, x)-\nu\left(T(t, x)-T_{0}\right)+q_{v}
$$


according to [3], page 149. Here

1. $T(x)$ is the absolute wire temperature at $x$, and $T_{0}$ the constant environment temperature

2. $q_{v}$ is the power volumetric density generated inside the wire

3. $k=\frac{\chi}{\rho c_{p}}$ is the thermal diffusivity of wire's material

4. $\nu=\frac{H p}{\rho c_{p} \omega}$, where $H$ is the surface coefficient heat transfer of the Newton linear cooling law.

The last equation is asserting that the conduction takes place in the wire only along $x$ and then not radially; and the system looses heat to its surrounding at a rate proportional to the temperature difference (Newton's cooling law): what is likely only with a small difference. If this is not, the heat lost by radiation shall be taken in account: from the wire's length $\mathrm{d} x$, the radiated power is: $\tilde{\sigma} p\left(T^{4}-T_{0}^{4}\right) \mathrm{d} x$, where $\tilde{\sigma}$ is a constant. In ideal conditions, we would have only the Stefan-Boltzmann constant

$$
\sigma=5.669 \times 10^{-8} W^{-2}{ }^{0} K^{-4}
$$

as area multiplier. With a real body, a factor $<1$ involving the emissivity, will relate the radiation of the real grey surface to that of the ideal black one. In addition, not the whole heat radiated by the wire will reach the environment. Then $\sigma$ has to be multiplied by the emissivity and by the view factor to give $\tilde{\sigma}$. Furthermore, in steady state $\partial T(t, x) / \partial t=0$, so that our termal stationary balance becomes an ordinary differential equation

$$
T^{\prime \prime}(x)-\frac{2 \tilde{\sigma}}{\chi R}\left(T^{4}(x)-T_{0}^{4}\right)+\frac{q_{v}}{\chi}=0
$$

where the symbol " means the second derivative operator $\mathrm{d}^{2} / \mathrm{d} x^{2}$. The heat will then be transmitted along the wire, but simultaneously, at each $x$ a part of it will be radiated outwards. The model is more accurate for very thin wires, and therefore certainly not for rods, whose radial dependence of $T$ has been ignored. Equation (1) displays a nonlinear, one-dimensional, steady model of both conduction and radiation through and from a indefinite wire, whose boundary at $x=0$ is kept at the surrounding temperature $T_{0}$.

The most straightforward BC for a problem like this, is to specify the temperature at the boundary, a Dirichlet-type BC. It will be recalled here that there is a second $\mathrm{BC}$ assigning somewhere heat flux (proportional to the gradient $\partial T / \partial x$, according to Fourier law) and called a Neumann-type BC. 
The second condition concerns $V_{0}$, namely the thermal gradient at $x=0$

$$
T^{\prime \prime}(x)-\frac{2 \tilde{\sigma}}{\chi R}\left(T^{4}(x)-T_{0}^{4}\right)+\frac{q_{v}}{\chi}=0, \quad T(0)=T_{0}, \quad T^{\prime}(0)=V_{0} .
$$

Now we impose both a Dirichlet and a Neumann boundary condition at $x=0$. We are, in such a way, formally faced with a Cauchy initial value problem like in particle Mechanics: the above ODE is modelling a undamped, quartic, steady, forced, one dimensional oscillator physically behaving as our conducting/radiating wire in thermal equilibrium. Likewise, the prescribed levels of temperature $\left(T_{0}\right)$ and gradient $\left(V_{0}\right)$ at boundary $x=0$ can be read as IC of position and speed.

\section{Thermal System ODE Integration}

The integration of (2) in general case of both non-zero and independent left boundary conditions $V_{0}$ and $T_{0}$, leads to a hyperelliptic integral with a nonhomogeneous $5^{\text {th }}$ degree polynomial under square root. In a particular, meaningful case we will give it in terms of the Gauss hypergeometric function. For the sake of convenience, let us rewrite (2)

$$
T^{\prime \prime}(x)=\frac{5}{2} a^{4} T^{4}(x)-\frac{1}{2} b^{4}, \text { where } a=\left(\frac{8 \tilde{\sigma}}{5 \chi R}\right)^{1 / 4}, b=2\left(T_{0}+\frac{q_{\nu}}{\chi}\right)^{1 / 4} .
$$

We introduce a supplementary constraint between the left boundary data $T_{0}$ and $V_{0}$

$$
\mathcal{L}:=V_{0}^{2}-T_{0}\left(a^{4} T_{0}^{4}-b^{4}\right)=0 .
$$

Such a constraint has been deliberately set for obtaining an integrable case: should be $\mathcal{L} \in \mathbb{R}, \mathcal{L} \neq 0$, the stationary distribution of $T$ with $x$ along the wire would be different, and would require a more difficult integration, as we have already said. Then we state the Theorem

Theorem 1. The solution $T(x)$ of initial value second order nonlinear problem:

$$
T^{\prime \prime}(x)=\frac{5}{2} a^{4} T^{4}(x)-\frac{1}{2} b^{4}, \quad T(0)=T_{0}>0, \quad T^{\prime}(0)=\sqrt{a^{4} T_{0}^{5}-b^{4} T_{0}}
$$

is defined for

$$
0 \leq x<x_{\infty}:=\frac{2}{3 a^{2} T_{0}^{3 / 2}}{ }_{2} F_{1}\left(\frac{3}{8}, \frac{1}{2} ; \frac{11}{8} ; \frac{b^{4}}{a^{4} T_{0}^{4}}\right) .
$$




\section{Furthermore}

$$
\lim _{x \uparrow x_{\infty}} T(x)=\infty .
$$

Finally, the inverse function $x(T)$ of the solution of (4) is given by

$$
x(T)=x_{\infty}-\frac{2}{3 a^{2} T^{3 / 2}}{ }_{2} F_{1}\left(\frac{3}{8}, \frac{1}{2} ; \frac{11}{8} ; \frac{b^{4}}{a^{4} T^{4}}\right) .
$$

For the proof of Theorem 1, we need to state in advance a definite hyperelliptic integration formula, as given in Theorem 2. Formula (7) allows to evaluate a hyperelliptic integral by means of functions $\Gamma$ and ${ }_{2} F_{1}$. It is a new, as far as we are concerned, and seems to own an intrinsic mathematical interest beyond the frame of its birth.

Theorem 2. If $u>1$, then

$$
\int_{1}^{u} \frac{d \tau}{\sqrt{\tau\left(\tau^{4}-1\right)}}=\frac{\sqrt{\pi}}{4} \frac{\Gamma\left(\frac{3}{8}\right)}{\Gamma\left(\frac{7}{8}\right)}-\frac{2}{3 u \sqrt{u}}{ }_{2} F_{1}\left(\frac{3}{8}, \frac{1}{2} ; \frac{11}{8} ; \frac{1}{u^{4}}\right) .
$$

Proof: Integrating term by term and making use of the Pochhammer symbol $(\cdot)_{n}$, we find

$$
\int_{1}^{u} \frac{d \tau}{\sqrt{\tau\left(\tau^{4}-1\right)}}=\frac{1}{4} \sum_{n=0}^{\infty} \frac{\left(\frac{1}{2}\right)_{n}}{\left(n+\frac{3}{8}\right) n !}-\frac{1}{4 u \sqrt{u}} \sum_{n=0}^{\infty} \frac{\left(\frac{1}{2}\right)_{n} u^{-4 n}}{\left(n+\frac{3}{8}\right) n !} .
$$

We ought to prove the convergence of the two series at right hand side of (8). The former is a numerical series convergent by the Raabe criterion as if

$$
x_{n}=\frac{\left(\frac{1}{2}\right)_{n}}{\left(n+\frac{3}{8}\right) n !} \quad \text { then we have } \quad \lim _{n \rightarrow \infty} n\left(\frac{x_{n}}{x_{n+1}}-1\right)=\frac{3}{2} .
$$

The latter converges, having the same general coefficient of the previous one, multiplied by $u^{-1}<1$. Both series can be evaluated through the hypergeometric function ${ }_{2} F_{1}$. In fact, we have first to observe that

$$
\frac{\left(\frac{3}{8}\right)_{n}}{\left(\frac{11}{8}\right)_{n}}=\frac{\frac{3}{8}}{n+\frac{3}{8}}
$$

Furthermore, recalling the definition of hypergeometric series

$$
{ }_{2} F_{1}(p, r ; s ; z)=\sum_{n=0}^{\infty} \frac{(p)_{n}(r)_{n}}{(s)_{n}} \frac{z^{n}}{n !}
$$


we obtain

$$
\frac{1}{4} \sum_{n=0}^{\infty} \frac{\left(\frac{1}{2}\right)_{n}}{\left(n+\frac{3}{8}\right) n !}=\frac{1}{4} \sum_{n=0}^{\infty} \frac{\left(\frac{1}{2}\right)_{n}\left(\frac{3}{8}\right)_{n}}{\frac{3}{8}\left(\frac{11}{8}\right)_{n} n !}=\frac{2}{3}{ }_{2} F_{1}\left(\frac{3}{8}, \frac{1}{2} ; \frac{11}{8} ; 1\right) .
$$

Taking the Gauss formula into account, see for instance [5], page 40

$$
{ }_{2} F_{1}(p, r ; s ; 1)=\frac{\Gamma(s) \Gamma(s-p-r)}{\Gamma(s-p) \Gamma(s-r)}
$$

we get

$$
{ }_{2} F_{1}\left(\frac{3}{8}, \frac{1}{2} ; \frac{11}{8} ; 1\right)=\frac{3}{8} \sqrt{\pi} \frac{\Gamma\left(\frac{3}{8}\right)}{\Gamma\left(\frac{7}{8}\right)}
$$

and therefore

$$
\int_{1}^{u} \frac{d \tau}{\sqrt{\tau\left(\tau^{4}-1\right)}}=-\frac{1}{4 u \sqrt{u}} \cdot \frac{8}{3}{ }_{2} F_{1}\left(\frac{3}{8}, \frac{1}{2} ; \frac{11}{8} ; \frac{1}{u^{4}}\right)+\frac{1}{4} \frac{8}{3} \frac{3}{8} \sqrt{\pi} \frac{\Gamma\left(\frac{3}{8}\right)}{\Gamma\left(\frac{7}{8}\right)}
$$

hereby we eventually obtain (7).

We can now proceed with the proof of Theorem 1 .

Proof of Theorem 1: The initial value problem (4) can be tackled by means of the classic Weierstraß method, see [7] or also [1], pages 287-292. Such autonomous equation, key of all our treatment, is

$$
T^{\prime \prime}(x)=f(x), \quad T(0)=T_{0}, \quad T^{\prime}(0)=V_{0}
$$

with the relation (3) between $T_{0}$ and $V_{0}$. Integrating we get

$$
x(T)=\int_{T_{0}}^{T} \frac{\mathrm{d} u}{\sqrt{\Phi(u)}}, \text { with } \Phi(T)=2 \int_{T_{0}}^{T} f(\xi) \mathrm{d} \xi+V_{0}^{2} .
$$

The sign's choice is due to the positivity of $T^{\prime}(0)=V_{0}$, see (4), moreover we have $\Phi(T)=a^{4} T^{5}-b^{4} T$. Henceforth we find the expression for the inverse function $x(T)$ of the solution $T(x)$ of (4)

$$
x(T)=\frac{1}{a^{2}} \int_{T_{0}}^{T} \frac{\mathrm{d} \tau}{\sqrt{\tau^{5}-\left(\frac{b}{a}\right)^{4} \tau}} .
$$

This implies that the problem is then meaningful for $T, T_{0} \geq b / a$. Moreover notice that the inversion $x(T) \longleftrightarrow T(x)$ is ensured by the construction of $x(T)$ itself

$$
x^{\prime}(T)=\frac{1}{a^{2} \sqrt{T^{5}-\left(\frac{b}{a}\right)^{4} T}}>0 .
$$


Finally, the integral in (9) for $T \rightarrow \infty$ converges to a value $x_{\infty}$, and this means that the inverse function $T(x)$ is unbounded for $x \uparrow x_{\infty}$. After a change of variable (9) becomes

$$
x(T)=\frac{1}{a^{2}}\left(\frac{a}{b}\right)^{3 / 2} \int_{\frac{a}{b} T_{0}}^{\frac{a}{b} T} \frac{\mathrm{d} \tau}{\sqrt{\tau\left(\tau^{4}-1\right)}}
$$

and, using Lemma 2, formula (7), we obtain

$$
x(T)=\frac{2}{3 a^{2}}\left[\frac{1}{T_{0}^{3 / 2}}{ }_{2} F_{1}\left(\frac{3}{8}, \frac{1}{2} ; \frac{11}{8} ; \frac{b^{4}}{a^{4} T_{0}^{4}}\right)-\frac{1}{T^{3 / 2}}{ }_{2} F_{1}\left(\frac{3}{8}, \frac{1}{2} ; \frac{11}{8} ; \frac{b^{4}}{a^{4} T^{4}}\right)\right]
$$

i.e., (6). The final step is proving (5), which follows from (6), by taking into account that

$$
\lim _{T \rightarrow \infty}{ }_{2} F_{1}\left(\frac{3}{8}, \frac{1}{2} ; \frac{11}{8} ; \frac{b^{4}}{a^{4} T^{4}}\right)=1 .
$$

We close this section by depicting graphics in the $(x, T(x))$ plane, of a testsolution that we obtained by our final formula (6) for reasonable values of $a, b, T_{0}$. For getting the local wire temperature as a function of $x$, it is enough to ask Mathematica ${ }^{\circledR}$ to plot the inverse function of $x(T)$ as expressed by our equation (6). The picture below is produced by taking $T_{0}=11, a=1 / 10, b=1$.

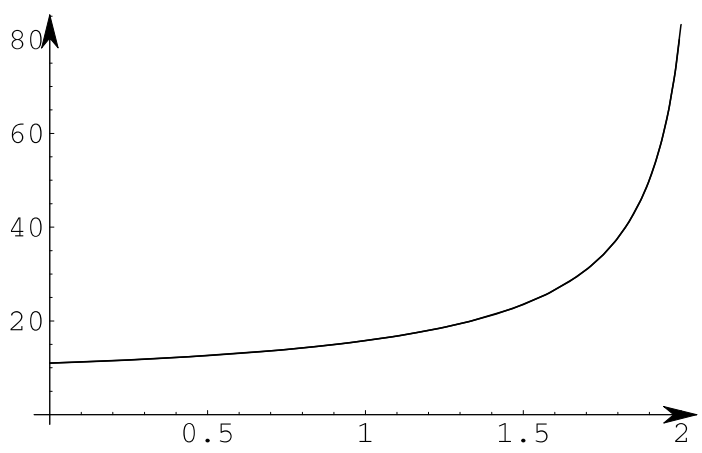

Figure 1: Overlap between numerical output from [6] and exact solution from (6)

By comparing our exact solution with the output of a numerical high performance method [6], a complete and minute overlap is confirmed.

\section{Conclusions}

The mixed equilibrium heat transmission consisting of a $x$-conduction through a indefinite wire with inner source is studied, assuming its boundary $x=0$ isother- 
mal with the environment which all the wire is radiating to. The Cauchy problem with thermal level and gradient at $x=0$ assigned independently would lead to an hyperelliptic integral which cannot be evaluated in an easy way. A supplementary constraint between the $x=0$ boundary data $T_{0}$ and $V_{0}$, see (3), is therefore introduced so that the resulting integral, even if hyperelliptic yet, can be expressed by Gauss hypergeometric function ${ }_{2} F_{1}$. On the contrary, the other constraint: $T, T_{0} \geq b / a$ comes from the problem itself for ensuring the reality of (9). The relevant solution has been found to be monotonically increasing and unbounded with a vertical asymptote, see Theorem 1 . Then the initial assumption about the indefiniteness of our wire shall be set aside: there is a length's limit $x_{\infty}$, depending on $T_{0}$ and $a$ and $b$, whose physical meaning is the following.

At a short distance from $x=0$, the thermal increase is low, being nearly all the generated power lost by radiation. But for high temperatures we have $T^{\prime}(x) \sim$ $O\left(T^{5 / 2}\right)$, with $T$ increasing more than exponentially: so that $T$ will grow more and more, and radiation will be not capable of dissipating both conducted and produced power. As a consequence, at a certain $x_{\infty}$, wire temperature suddenly will jump unbounded, and our problem will have a smooth, monotonic and bounded namely physically acceptable - solution for $0 \leq x<x_{\infty}$.

\section{Acknowledgements}

Research supported by MIUR grant: Equazioni Differenziali e Problemi Geometrici.

\section{References}

[1] Agostinelli C. and Pignedoli A., Meccanica Razionale, vol. 2, Zanichelli, Bologna, 1978.

[2] Baumeister K. and Hamill T., Hyperbolic Heat-conduction Equation - a Solution for the Seminfinite Body Problem, Journal of Heat Transfer 91 (1969) 543-548.

[3] Carslaw H. and Jaeger J., Conduction of Heat in Solids, Clarendon Press, Oxford, 1959.

[4] Morse, M. and Feshbach H., Methods of Theoretical Physics, McGraw-Hill Book Company, New York, 1953.

[5] Magnus W., Oberhettinger F. and Soni R., Formulas and Theorems for the Special Functions of Mathematical Physics, Springer, Berlin 1966. 
[6] Schwalbe D. and Wagon S., VisualDSolve, Springer, New York, 1997.

[7] Weierstraß K., Über eine Gattung reell periodischer Funktionen, In: Mathematische Werke II, Mayer \& Müller, Berlin, 1895.

Giovanni Mingari Scarpello

Facoltà di Economia

Libera Università di Bolzano

via Sernesi, 1, 39100 Bolzano, Italy

E-mail address:

GMingariScarpello@unibz.it

Arsen Palestini

Dipartimento di Matematica Ulisse Dini

viale Morgagni, 67/a, 50134 Firenze, Italy

E-mail address:

palestini@math.unifi.it

Daniele Ritelli

Dipartimento di Matematica

per le Scienze Economiche e Sociali

viale Filopanti, 540126 Bologna, Italy

E-mail address:

dritelli@economia.unibo.it 
